\title{
Soziale Beziehungen, soziales Kapital und soziale Netzwerke - eine begriffliche Einordnung
}

\section{Nico Vonneilich}

\section{Überblick}

- Es werden die Begriffe soziale Unterstützung, soziales Kapital, soziale Kohäsion und soziales Netzwerk definiert und voneinander abgegrenzt.

- Überschneidungen und Eigenheiten der jeweiligen Konzepte werden herausgearbeitet und sollen $\mathrm{zu}$ einer begrifflichen Präzision und $\mathrm{zu}$ einem besseren Verständnis des Forschungsfeldes beitragen.

- Soziale Netzwerke lassen sich sowohl auf Ebene einzelner Individuen (Mikro-) als auch auf Ebene von Gruppen und Gemeinden (Meso-/ Makro-Ebene) beschreiben.

- Theorien des sozialen Netzwerks beinhalten sowohl quantitative Aspekte sozialer Beziehungen (Struktur) als auch qualitative Aspekte (Funktion).

\section{$1 \quad$ Einleitung und Hintergrund}

Soziale Beziehungen sind ein Kerngebiet der Soziologie, ihr Grundgerüst. Ohne soziale Beziehungen entstehen keine gesellschaftlichen Zusammenhänge. Die Untersuchung sozialer Beziehungen kann auf eine lange Forschungstradition zurückblicken und diese wird weiter fortgeführt, in stetig sich differenzierenden

\footnotetext{
N. Vonneilich $(\bowtie)$

Institut für Medizinische Soziologie, Universitätsklinikum Hamburg-Eppendorf, Hamburg, Deutschland

E-Mail: n.vonneilich@uke.de
} 
und spezialisierenden Subsystemen. Ziel des vorliegenden Kapitels ist es, einen kurzen Überblick über die Forschungstraditionen zu sozialen Beziehungen zu geben. Dabei soll es insbesondere um eine begriffliche Klärung und, wo möglich, auch um eine Abgrenzung verschiedener Konzepte voneinander gehen, die im Laufe der Beschäftigung mit sozialen Beziehungen in der Soziologie und anderen nahestehenden Disziplinen (etwa der Sozialpsychologie) entwickelt wurden. Warum ist eine solche Abgrenzung und Einordnung notwendig? In der Auseinandersetzung mit Forschungsarbeiten rund um das Thema soziale Beziehungen kann festgestellt werden, dass unterschiedliche Begriffe synonym verwendet werden und ursprünglich intendierte Abgrenzungen untereinander mit der Zeit verschwimmen. Das vorliegende Kapitel orientiert sich an den folgenden Leitfragen: Welche Begrifflichkeiten gibt es in der soziologischen Forschung zu sozialen Beziehungen? Wie werden diese definiert? Und wie kann aus diesen unterschiedlichen Begriffen ein übergreifendes Konzept zu sozialen Netzwerken entstehen? Das Forschungsfeld zu sozialen Beziehungen ist breit gefächert und ist Thema vieler Disziplinen. Es reicht von der Soziologie hin zur Sozialpsychologie, von Ökonomie über Public Health hin zur Epidemiologie, ohne dass dies eine vollständige Liste aller Fächer und Forschungsschwerpunkte sein soll, die sich mit diesem Thema befassen. Die wissenschaftliche Betrachtung sozialer Beziehungen hat in der Soziologie eine längere Geschichte: diese erstreckt sich von Durkheims bekannter Arbeit zum Selbstmord (Durkheim 1993, Original 1897), über Parsons funktionalistisch ausgerichtete Gesellschaftsanalyse (Parsons 1951), welche den sozialen Interaktionen zugrunde liegenden Werten und Normen Beachtung schenkt, bis hin zu Bourdieus Kapital-Theorien und seinen Analysen der gesellschaftlichen Differenzierung (Bourdieu 1994). Die dabei entstandene begriffliche Vielfalt ist durchaus gewünscht und zeugt von der Pluralität an Forschungsaktivitäten. Diese reicht vom sozialen Kapital über soziale Kohäsion hin zu sozialen Netzwerken. Gleichzeitig aber ist eine solche Ausdifferenzierung problematisch, wenn die Begriffe und die dahinterstehenden Konzepte sich überschneiden oder synonym verwendet werden, ohne dass damit klare Definitionen und Abgrenzungen verbunden sind (Holt-Lunstad et al. 2010; Berkman und Krishna 2014). Berkman und Glass fassen dies wie folgt prägnant zusammen: „When investigators write about the impact of social relationships on health, many terms are used loosely and interchangeably, including social networks, social support, social ties and social integration" (Berkman und Glass 2000, S. 137). Ziel der folgenden Übersicht ist es, die Begriffe zu ordnen und voneinander abzugrenzen, deren Entstehung zu skizzieren, um darauf aufbauend Zusammenhänge mit Gesundheit beschreiben und diskutieren zu können.

Soziale Beziehungen dienen im Rahmen des vorliegenden Kapitels als Oberbegriff für eine ganze Reihe unterschiedlicher Konzepte zur Beschreibung des 
sozialen Miteinanders in Gesellschaften. Dabei können unterschiedliche Merkmale differenziert werden. So lassen sich grundsätzlich die Quantität und die Qualität sozialer Beziehungen unterscheiden. Unter Quantität ist die Anzahl unterschiedlicher sozialer Kontakte oder die Häufigkeit sozialer Kontakte zu verstehen. Jedem sozialen Kontakt kann eine bestimmte Qualität zugeschrieben werden. Wie sind diese Kontakte beschaffen oder welche Ressourcen sind über diese Kontakte erreichbar? Soziale Kontakte sind Vorbedingung für den Austausch von Ressourcen und sozialer Unterstützung. Jedoch kann nicht zwingend davon ausgegangen werden, dass eine steigende Zahl sozialer Kontakte mit einer zunehmenden Verfügbarkeit von Ressourcen und Unterstützungsleistungen einhergeht. Nicht jede Person innerhalb eines sozialen Gefüges ermöglicht Ressourcenzugang.

Eine andere Unterscheidungsmöglichkeit ergibt sich durch die Ebene, auf der soziale Beziehungen eingeordnet werden können. Während auf der Mikro-Ebene soziale Beziehungen einer Einzelperson untersucht werden, sind es auf der MesoEbene (institutionelle) Netzwerke beispielsweise von Gemeinden, Kommunen, Stadtteilen oder Schulen, und entsprechend auf der Makro-Ebene Länder und Staaten. Letzteres wird häufig auf Basis von Indikatoren für Vertrauen, Normen oder staatliche Institutionen gemessen, worauf im Folgenden noch eingegangen wird.

Ausgehend von dieser grundlegenden Differenzierung sollen im Folgenden zentrale Begriffe wie soziale Unterstützung, soziales Kapital, soziale Kohäsion und soziale Netzwerke eingeführt und erörtert werden.

\section{$2 \quad$ Klärung der Begrifflichkeiten}

\subsection{Soziale Unterstützung}

Zentrale qualitative Dimension auf der Mikro-Ebene sozialer Beziehungen ist die soziale Unterstützung. Ohne soziale Kontakte, ohne eine Einbettung in soziale Netzwerke kann keine soziale Unterstützung stattfinden. Sind Anzahl und Frequenz sozialer Kontakte Maße für die Quantität sozialer Beziehungen, dann ist soziale Unterstützung ein $\mathrm{Ma}$ für die Qualität dieser Beziehungen. In der Unterstützungsforschung wird zwischen objektiven und subjektiven Aspekten von Unterstützung unterschieden (Turner und Marino 1994). Es konnte gezeigt werden, dass nicht nur die tatsächlich erhaltene Unterstützung relevant ist, sondern die subjektiv wahrgenommene Unterstützung eine zentrale Rolle spielt, gerade im Zusammenhang mit (mentaler) Gesundheit. Bereits das Gefühl, im Ernstfall über soziale Unterstützung zu verfügen, kann negative Auswirkungen von akutem 
Stress abmildern, ohne dass tatsächlich Unterstützung in Anspruch genommen wurde (Cobb 1976; House et al. 1988; Turner und Marino 1994; Uchino 2009). Zudem wurden zwischen tatsächlich erhaltener und subjektiv wahrgenommener Unterstützung nur geringe Zusammenhänge gefunden, was darauf hindeutet, dass diese als jeweils relativ eigenständige Konstrukte angesehen werden können (Barrera 1986; Lakey und Cohen 2000). Dieser funktionale Aspekt sozialer Beziehungen umfasst in der Regel Unterstützungsformen auf emotionaler, instrumenteller sowie informationeller Ebene.

Emotionale Unterstützung bezieht sich auf diejenigen sozialen Kontakte, die für Gespräche über eigene Gefühle zur Verfügung stehen, die zur Diskussion alltäglicher Ängste und Sorgen beitragen oder auch Bestätigung für Sympathie und Zuneigung bieten können (siehe Tab. 1, dazu auch Lin et al. 1999). Instrumentelle Unterstützung dagegen umfasst diejenigen Unterstützungsformen, die sich durch praktische Hilfe - beispielsweise im Haushalt, mit der Kinderbetreuung oder durch das Leihen von Geld oder anderen Waren - auszeichnen. Unter informationeller Unterstützung werden all jene Leistungen erfasst, die Wissen zum Lösen bestimmter Probleme, oder Wissen über den Zugang zu bestimmten Ressourcen wie beispielsweise innerhalb von Gemeinden, verfügbar machen. In Tab. 1 findet sich eine kurze Darstellung unterschiedlicher Aspekte sozialer Unterstützung, basierend auf Wills und Shinar (2000).

Ein wichtiges Grundprinzip, auf dessen Basis es zu unterschiedlichen Formen sozialer Unterstützung kommen kann, ist die soziale Reziprozität (Siegrist und Wahrendorf 2016). Dieses besagt, dass Menschen für eine bestimmte erbrachte Leistung auch eine entsprechende Gegenleistung erwarten (Prinzip der Gegenseitigkeit). Diese Erwartung macht viele Formen der sozialen Interaktion überhaupt erst möglich. Eine Gegenleistung muss nicht dieselbe Form wie die erhaltene Leistung haben, sollte aber als adäquat und ähnlich empfunden werden. Leistet man beispielsweise Bekannten Hilfe bei einem Umzug (instrumentelle Unterstützung), so kann erwartet werden, bei Bedarf eine ähnliche Leistung zu erhalten. Werden diese Erwartungen nicht erfüllt, wird die Reziprozitätsnorm verletzt und dauerhafter sozialer Austausch weniger wahrscheinlich. Je nachdem, in welcher Beziehung man sich mit anderen Personen befindet, kann sich die Reziprozitätsnorm verändern. Zum Beispiel ist man innerhalb der Familie, für enge Freunde oder Verwandte eher bereit, eine Leistung zu erbringen, ohne eine zeitlich (un-)mittelbare entsprechende Gegenleistung zu erwarten. Die generalisierte Reziprozität meint, dass nicht einzelne erbrachte Leistungen jeweils entsprechend erwidert werden. Vielmehr kann ein generelles reziprokes Verhalten zu einem deutlich späteren Zeitpunkt erwartet werden. Klassisches Beispiel hierfür wäre die Eltern-Kind Beziehung, in der Eltern Unterstützung für ihre Kinder 


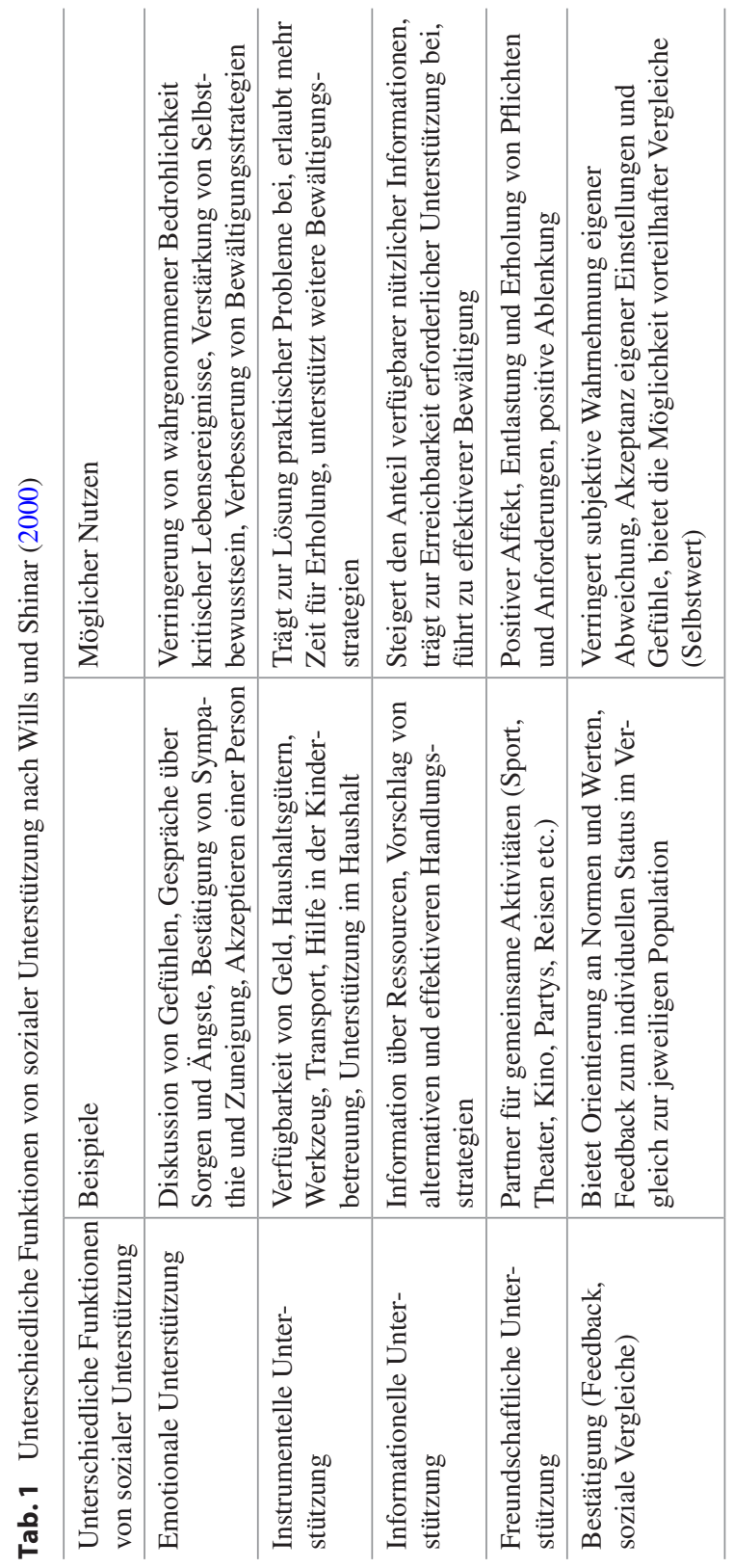


leisten, ohne dass diese jeweils direkt erwidert werden. Dafür erwarten Eltern möglicherweise im höheren Lebensalter eine entsprechende Unterstützung durch die Kinder. Der Forschungsansatz zu sozialer Unterstützung fokussiert stark auf die individuelle Ebene, sie fragt nach Unterstützungsformen, welche Einzelnen zur Verfügung steht. Da jedoch durch die Konzentrierung auf Einzelne der strukturelle Aspekt aus dem Blick gerät, wurde wiederholt von der Wissenschaft vorgeschlagen, zusätzlich die Gesamtstruktur der Netzwerke zu untersuchen, um die strukturelle Bedingtheit von individuellen Unterstützungsleistungen und -möglichkeiten herausarbeiten zu können (Holt-Lunstad 2010; Berkman 2014). Hier stehen Fragen im Vordergrund, wie soziale Beziehungsgefüge geschaffen sein müssen, damit soziale Unterstützung stattfinden kann. Welche Faktoren innerhalb sozialer Beziehungen bedingen soziale Unterstützung, welche wirken eher hinderlich? Lassen sich strukturelle Merkmale von sozialer Unterstützung identifizieren? Dies vermag eine zu sehr auf die individuelle Unterstützung fokussierte Analyse nur schwer zu beantworten.

\subsection{Soziales Kapital}

Soziales Kapital als Untersuchungsgegenstand lässt sich in verschiedenen Fachdisziplinen (Soziologie, Ökonomie, Sozialpsychologie, Politikwissenschaft) verorten. Als zentrale Gemeinsamkeiten unterschiedlicher Konzepte von sozialem Kapital lassen sich dabei die Folgenden ausmachen (Berkman und Krishna 2014; Kawachi und Berkman 2014). Soziales Kapital wird als Ressource verstanden (1), welche nicht durch Einzelne produziert wird, sondern erst durch soziale Interaktion mit Anderen entsteht (2). In der Soziologie lassen sich relativ grob zwei Forschungstraditionen unterscheiden. Auf der einen Seite eine eher französische Forschungstradition, insbesondere geprägt durch die Arbeiten von Pierre Bourdieu. Auf der anderen Seite ein eher amerikanischer Forschungszweig, hier können insbesondere James Coleman und Robert Putnam genannt werden.

Der Kapitaltheorie nach Bourdieu zufolge steht neben ökonomischem Kapital auch kulturelles und soziales Kapital zur Verfügung. Soziales Kapital meint, dass über soziale Beziehungen Zugang zu Ressourcen ermöglicht werden kann (Bourdieu 1994). Auch können Individuen gezielt in diese sozialen Beziehungen investieren, um entsprechend Zugang zu sozialem Kapital zu erhalten, was sich wiederum auf die anderen Kapitalformen auswirken kann. Es wird von einer „,...] Konkurrenz zwischen Investitionen in Sozialkapital und andere Kapitalien [...]“" gesprochen (Lüdicke und Diewald 2007, S. 15). Soziales Kapital wird als 
Merkmal einzelner Individuen betrachtet, welche mit diesem oder durch dieses handeln können.

Die Ansätze von Coleman (1990) und Putnam unterscheiden sich in ihrer Herangehensweise von Bourdieu insofern, als soziales Kapital stärker als Eigenschaft von sozialen Netzwerken verstanden wird und dementsprechend die Betonung auf der zwischenmenschlichen Ebene liegt. „Unlike other forms of capital, social capital inheres in the structure of relations between actors and among actors" (Coleman 1990, S. 98). Diese nordamerikanische Tradition entwickelt ihr Verständnis von sozialem Kapital aus der Untersuchung sozialer Netzwerke. Im Vordergrund steht dabei die Frage, wie und unter welchen Bedingungen sich soziales Kapital in sozialen Netzwerken ausbildet (Lin 2000).

Betrachtet man soziales Kapital auf der Mikro-Ebene der Individuen, so ergeben sich Überschneidungen sowohl mit dem Konzept der sozialen Unterstützung als auch mit sozialen Netzwerken. Soziale Unterstützung geht von zumeist engen, eher stärkeren sozialen Beziehungen aus. Das Konzept des sozialen Kapitals hingegen unterscheidet zwischen starken und schwachen Beziehungen und sieht gerade die Möglichkeiten der schwächeren Beziehungen, neue Informationen und Ressourcen zur Verfügung zu stellen und ist hier eng an das Konzept sozialer Netzwerke angelehnt. Dies wurde unter dem Schlagwort strength of weak ties diskutiert (Granovetter 1973; Putnam 1995). Diese von Granovetter entwickelte These besagt, dass gerade nicht die häufig frequentierten, engen und mit höherer Intensität geführten Beziehungen (auch als bonding social capital bezeichnet) relevante Ressourcen, wie etwa Zugang zum Arbeitsmarkt, zur Verfügung stellen können, sondern dass dies durch Kontakte ermöglicht werden kann, welche sich durch geringere Kontaktfrequenzen und niedrige Intensität charakterisieren lassen. Diese als bridging social capital (überbrückendes soziales Kapital) bezeichneten Kontakte zeichnen sich dadurch aus, dass sie über unterschiedliche soziale Gruppen hinweg etabliert werden und dass sie die Zugangswahrscheinlichkeit zu bestimmten Ressourcen erhöhen (Lin et al. 1999). Demgegenüber sind es die engeren Kontakte, die eher instrumentelle und emotionale Unterstützung zur Verfügung stellen (Dahl et al. 2010).

Auf der Meso- oder auch der Makro-Ebene betrachtet, lässt sich soziales Kapital zugleich als Eigenschaft sozialer Gruppen sowie als Charakteristik von Lebensräumen bestimmen. Aufbauend auf der Erfassung individueller Einschätzungen wie etwa der Reziprozität und dem Vertrauen in die jeweilige Lebensumgebung (z. B. Nachbarschaft, Stadtteil), zu sozialem und ehrenamtlichem Engagement sowie generellen Einstellungen zu Gruppen oder Lebensräumen werden Indizes auf einer Aggregatebene gebildet, welche das Ausmaß an 
sozialem Kapital widerspiegeln und entsprechend als Charakteristik von Gruppen oder umgrenzten Räumen verwendet werden. Grundlegende Annahme ist hierbei, dass erst durch erlebte Reziprozität und Vertrauen sowie auf gemeinsam getragenen Werten und Normen regelmäßige Interaktion entsteht, welche wiederum Zugang zu Ressourcen innerhalb von Gruppen ermöglicht und soziales Kapital entstehen kann (Putnam 1995; Ichida et al. 2009; Dahl et al. 2010). Je höher entsprechend das Vertrauen in die eigene Lebensumgebung ist, desto wahrscheinlicher wird der Theorie zufolge auch die Entwicklung stabiler sozialer Beziehungsgefüge, in denen soziales Kapital verfügbar ist. Eine ganze Vielzahl von Studien konnte solche Zusammenhänge bestätigen (siehe Airaksinen et al. 2015; Pickett und Pearl 2001).

\subsection{Soziale Kohäsion}

Ein weiterer Begriff, der im Zusammenhang mit der Untersuchung von sozialen Beziehungen innerhalb umgrenzter Räume immer wieder genannt wird, ist soziale Kohäsion. Damit werden vornehmlich subjektive Einschätzungen zu Verbindungen von Mitgliedern innerhalb sozialer Gruppen beschrieben. Innerhalb jeder Gruppe findet sich ein gewisser Grad an sozialer Kohäsion. Grundsätzlich kann hierbei zwischen der strukturellen Kohäsion (structural cohesion) sowie dem Zugehörigkeitsgefühl der einzelnen Mitglieder (perceived cohesion, sense of togetherness) (Bollen und Hoyle 1990). Durch die Betonung des Zugehörigkeitsgefühls, welches implizit auch gemeinsam getragene Werte und Normen beinhaltet, schließt das Konzept der perceived social cohesion eng an das Konzept des sozialen Kapitals an.

Eine hohe strukturelle soziale Kohäsion meint, dass die Mitglieder einer bestimmten Gruppe untereinander eng verbunden sind. Die stärkste Kohäsion innerhalb einer Gruppe ist demnach erreicht, wenn jedes Mitglied eines Netzwerks direkt mit jedem anderen Mitglied verbunden ist, eine geringe Kohäsion, wenn viele Mitglieder eines Netzwerks nur lose und indirekt miteinander verbunden sind. Die subjektive Kohäsion muss nicht der strukturellen Kohäsion entsprechen, denn diese können voneinander abweichen. Die soziale Kohäsion wird als ein Merkmal von Gruppen oder räumlichen Gebieten umschrieben und lässt sich daher der Meso- und Makro-Perspektive von sozialen Beziehungen zuordnen.

Soziale Kohäsion wird häufig anhand subjektiver Einschätzungen gemessen. Dabei stehen individuelle Einschätzungen und Wahrnehmungen zu den jeweiligen Gruppen im Vordergrund sowie tatsächliche Aktivitäten der Individuen innerhalb 
der Gruppen. Eine starke Kohäsion innerhalb von sozialen Gruppen geht häufiger mit höherer sozialer Kontrolle nach innen einher, während solche Gruppen nach außen relativ geschlossen bleiben (Kawachi und Berkman 2014). Beispiele solcher kohäsiver Gruppen finden sich unter Immigranten, die aufgrund ihrer ethnischen Zugehörigkeit starke Bindungen untereinander aufbauen, z. B. aufgrund sprachlicher Barrieren, aber nach außen relativ geschlossen bleiben. Ähnliches gilt für hoch vernetzte Nachbarschaften oder dörfliche Gemeinschaften.

Das Konzept der perceived social cohesion wurde kritisiert, weil es im Vergleich zum sozialen Kapital viele Überschneidungen aufweist und klare Alleinstellungsmerkmale für dieses Konzept fehlen. Da jedoch die Erfassung insbesondere von umfangreichen Strukturen des sozialen Kapitals in Gemeinden oder Nachbarschaften sehr aufwendig und schwer durchführbar ist, kann wahrgenommene soziale Kohäsion als eine alternative Form der Erfassung begriffen werden und erhält seine Berechtigung durch die methodische Umsetzbarkeit, weniger aufgrund der theoretischen Verortung.

Beide Begriffe, sowohl soziales Kapital als auch soziale Kohäsion, bergen die Möglichkeit negativer Prozesse innerhalb von sozialen Gruppen. Dazu gehören soziale Exklusion, Stigmatisierung, Diskriminierung und andere negative Effekte sozialer Beziehungen wie etwa andauernde Konflikte. Ein hoher Grad an sozialer Kohäsion und eine enge Vernetzung von Mitgliedern untereinander schließt auch die Möglichkeit der Exklusion von Nichtmitgliedern mit ein (siehe dazu Kawachi und Berkman 2014). Zudem tendieren Gruppen mit hoher sozialer Kohäsion dazu, wenig Kontakt zu anderen Gruppen zu haben. Kontakte zwischen unterschiedlichen Gruppen können positive Einstellungen gegenüber Anderen unterstützen, ebenso werden so eher Vorurteile und negative Einstellungen abgebaut. Dies wurde insbesondere im Kontext unterschiedlicher ethnischer Gruppen untersucht (Laurence und Bentley 2016; Hewstone 2015) und im Lichte zweier unterschiedlicher Theorien dazu diskutiert (conflict theory Putnam 2007, intergroup contact theory Brown und Hewstone 2005). Mangelnde soziale Kontakte zwischen Gruppen können entsprechend negative Einstellungen fördern. Weitere Ausführungen zu negativen Aspekten sozialer Beziehungen finden sich im Kap. „Negative Beziehungsaspekte und gesundheitliche Ungleichheiten“.

\subsection{Soziale Netzwerke}

Abschließend soll im Folgenden der Begriff der sozialen Netzwerke näher erläutert werden. Die soziologische Netzwerkanalyse interessiert sich für Beziehungen zwischen Individuen sowie für die Eigenschaften dieser 
Beziehungen, mehr als für die Eigenschaften der Individuen selbst. Grundgedanke ist, dass Individuen nicht unabhängig handeln, sondern dass diese Handlungen eingebettet sind in ein Netz zwischenmenschlicher Beziehungen (Burt 1982). Auch stehen nicht nur die Beziehung zwischen Ego, also dem zentralen Akteur, von dem die Betrachtung des Netzwerkes ausgeht, und verschiedenen Alteri, also Referenzpersonen von Ego im betrachteten Netzwerk, im Mittelpunkt, vielmehr geht es in der Netzwerkanalyse darum, ein gesamtes Beziehungsgeflecht zu untersuchen. Denn die Beziehung zwischen Ego und den Alteri wird auch beeinflusst durch Beziehungen der Alteri untereinander, die indirekt wiederum mit Ego verbunden sind. Soziale Interaktion und soziale Prozesse werden nicht allein durch Merkmale der Individuen, sondern durch ihre Integration in ein soziales Umfeld erklärbar (Häußling 2010). Um diese sozialen Zusammenhänge zu veranschaulichen, werden grafische Netzwerkmodelle entwickelt, die die Beziehungen sichtbar machen. Mit dem Blick der Netzwerkforschung verlässt man entsprechend schnell die Mikroperspektive der Einzelnen, hin zu einer Meso-Ebene, welche die Verflechtungen von Individuen und Gruppen sichtbar macht. Wichtiger Grundgedanke in der Netzwerkforschung ist es, dass nicht nur die Position eines Individuums innerhalb eines sozialen Netzwerks identifiziert werden kann, sondern durch Offenlegung der Strukturen eines Netzwerks auch die Möglichkeiten für Kontaktfähigkeit, Einfluss und Möglichkeiten der Kontrolle innerhalb von Netzwerken analysiert werden können. Diese Strukturen, welche sich anhand netzwerktheoretischer Begrifflichkeiten wie etwa Knoten, Dichte, Zentralität und Position beschreiben lassen, werden zur Beschreibung sozialer Phänomene hinzugezogen (Holzer 2009). Eine Erläuterung der unterschiedlichen Begrifflichkeiten findet sich im Kap. „Netzwerktheorie(n)“. Die Komplexität sozialer Netzwerke ergibt sich aus den verschiedenen möglichen Formen und Arten der Interaktion von Individuen und Gruppen untereinander.

Zudem lassen sich soziale Netzwerke nach ihrem jeweiligen Charakter unterscheiden, welcher formaler Natur sein kann, wenn es sich um Organisationen und Vereine handelt, oder informell, wenn es sich um persönliche, verwandtschaftliche oder freundschaftliche Netzwerke und Kontakte handelt. Weiterhin ist eine Differenzierung nach Frequenz, nach Intensität sowie nach Größe und Reichweite der Netzwerke, der Extension, möglich. Die frühe (soziologische) Netzwerkforschung hat sich vor allem auf diese eher quantitativen Aspekte sozialer Beziehungen konzentriert. Hier weist der Begriff der sozialen Netzwerke Überschneidungen mit dem Konzept der sozialen Integration auf. Einer Definition von Laireiter (1993) zufolge lässt sich soziale Integration als Integration von Individuen in soziale 
Gruppen, Vereine oder ehrenamtliche Organisationen, als die Anzahl sozialer Kontakte zu Familie, Verwandten und Freunden sowie die Verfügbarkeit und der Zugang zu sozialen und interpersonalen Ressourcen verstehen. Gleichzeitig verweist soziale Integration auf Normen und Werte als Orientierung für individuelle Handlungen, welche durch soziale Interaktion entstehen und durch diese verstetigt werden. Zahlreiche Indikatoren wurden entwickelt, um den Grad an sozialer Integration innerhalb sozialer Netzwerke zu erfassen. Beispielsweise lassen sich aus der sozialepidemiologischen Forschung der Social Integration Index von Berkman oder der Social Connection Index von Kaplan aufführen (Berkman und Syme 1979; Kaplan et al. 1977). In jüngerer Zeit wird nun auch in der Netzwerkforschung zunehmend versucht, das bisherige, eher quantitative Verständnis sozialer Netzwerke zu erweitern und auch qualitative Aspekte in die Erforschung sozialer Netzwerke einzubeziehen, indem beispielsweise die Verfügbarkeit von Ressourcen oder gemeinsam geteilte Normen und Werte innerhalb von Netzwerken mit in Betracht gezogen werden (Henning und Kohl 2011).

Was der soziologischen Untersuchung sozialer Netzwerke bisher weitgehend fehlt, ist eine eigenständige Theorie (Holzer 2009). Es finden sich Anknüpfungspunkte an Rational Choice-Ansätze sowie an strukturalistische Gesellschaftstheorien, auch in der Systemtheorie lassen sich Zusammenhänge zur Untersuchung von sozialen Netzwerken herstellen. In der Netzwerkforschung stehen formale und methodische Fragen im Vordergrund, weniger die Entwicklung einer gemeinsamen theoretischen Basis. In früheren Arbeiten wurde dieses Fehlen von übergeordneter Theorie auch als strukturelle Intuition der Netzwerktheorie bezeichnet (Freeman 2004). Holzer hebt dies in seinem Beitrag zur Netzwerktheorie wie folgt hervor: „Um den entscheidenden Schritt von der Netzwerkanalyse zu einer Netzwerktheorie machen zu können, muss jedoch den besonderen Konstitutionsbedingungen [der Netzwerkanalyse] Rechnung getragen werden " (Holzer 2009, S. 264). Diese Aussage bezieht sich darauf, dass bisherige Ansätze einer Netzwerktheorie in verschiedenen wissenschaftlichen Disziplinen Anklang finden, wie etwa der Physik, der Biologie, der Psychologie und der Soziologie, diese jeweiligen Ansätze aber nicht immer auf andere Bereiche übertragbar sind und eine gemeinsame theoretische Fundierung entsprechend erschweren. Es gibt bisher nur vereinzelt Ansätze, welche auf der Grundlage theoretischer Überlegungen versuchen, die relationale Analyse mit kulturellen und symbolischen Aspekten zu füllen, um so Handlungen und Interaktionen erklärbar zu machen (White 1995; Gibson 2005; Fuhse 2008). Ausführlicher auf die theoretischen Hintergründe und methodischen Aspekte der Netzwerkforschung wird im Kap. „Netzwerkanalyse“ eingegangen. 
Den Autorinnen und Autoren des vorliegenden Sammelbands liegt ein gemeinsames Verständnis sozialer Netzwerke zugrunde, welches sich von den bisher beschriebenen Begriffen abgrenzt beziehungsweise einzelne Aspekte unterschiedlicher Definitionen sozialer Beziehungen bewusst miteinbezieht. Demnach sind soziale Netzwerke zunächst einmal mehr als die sozialen Kontakte eines Individuums. Soziale Netzwerke heben sich dann von sozialer Integration $\mathrm{ab}$, wenn sie Aussagen über die Struktur sozialer Kontakte zulassen, also über die individuellen sozialen Kontakte auch die Vernetzung der Alteri untereinander sichtbar macht. Damit geht ein solcher Netzwerkbegriff über das Verständnis von Netzwerken wie etwa in den oben genannten Indizes (z. B. Social Network Index) deutlich hinaus. In Bezug auf das soziale Kapital grenzt sich der Begriff des sozialen Netzwerks insofern ab, als dass nicht nur beschrieben wird, welche Ressourcen oder Normen und Werte in bestimmten Gruppen verfügbar sind, sondern sich zudem darauf fokussiert, wie diese entstehen und reproduziert werden und welche Muster sozialer Netzwerke bestimmte Ressourcen ermöglichen oder verhindern. Ein solcher Netzwerkbegriff fragt nach der strukturellen Bedingtheit von sozialer Unterstützung und kann so die Forschung zu sozialer Unterstützung ergänzen, da diese mit einem Fokus auf individuelle Unterstützungsformen und -muster die soziale Struktur hinter der wahrgenommenen oder tatsächlichen Unterstützung vernachlässigt, wie eingangs gezeigt wurde. Soziale Kohäsion kann helfen, die Verbundenheit sozialer Netzwerke untereinander zu beschreiben und ist in dieser Funktion auch bereits in der Netzwerktheorie etabliert.

\section{$3 \quad$ Zusammenfassung und Ausblick}

Ziel des vorliegenden Beitrages ist es, die unterschiedlichen Begriffe in der Forschung zu sozialen Beziehungen zu klären und zu ordnen. Insbesondere in der Gesundheits- und der Medizin-Soziologie haben sich, teilweise unabhängig voneinander, teilweise eng miteinander verknüpft, gerade das soziale Kapital, soziale Netzwerke und soziale Unterstützung als Begrifflichkeiten in der Forschung rund um soziale Beziehungen etabliert. Ein immer wiederkehrender Vorwurf in diesem Forschungsbereich ist, dass Begrifflichkeiten unklar definiert oder auch synonym verwendet werden, was nicht zu einer Klärung der jeweiligen Begriffe beiträgt.

Aufbauend auf einer begrifflichen Klärung soll ein gemeinsamer Begriff von sozialen Netzwerken entwickelt werden. Ein umfängliches Verständnis sozialer Netzwerke umfasst sowohl quantitative als auch qualitative Aspekte sozialer 
Beziehungen. Zunächst repräsentieren soziale Netzwerke die Struktur, innerhalb welcher soziale Unterstützung und soziale Integration stattfinden können. Durch ihre Beschaffenheit ermöglichen oder verhindern soziale Netzwerke die Entstehung und die Verbreitung von sozialem Kapital. Anhand sozialer Netzwerke kann die strukturelle Bedingtheit sozialer Unterstützung gezeigt werden, in welchem Grad Individuen miteinander in Kontakt sind, wie weit diese Beziehungen reichen und wie dauerhaft diese ausgeprägt sind. Ebenso lassen sich qualitative Aspekte erfassen: die Intensität der Interaktionen, die verfügbaren Ressourcen zwischen Netzwerkmitgliedern oder auch die Normen und Werte und deren Entsprechung innerhalb von Netzwerken lassen Rückschlüsse auf die Qualität der Netzwerke zu. Insofern fließen in ein solches Verständnis auch Aspekte des sozialen Kapitals ein, in welchem postuliert wird, dass nicht nur die nahestehenden Kontakte Ressourcen zur Verfügung stellen, sondern dass dies insbesondere über schwache Verbindungen geschehen kann (weak ties).

Ebenso lassen sich Netzwerke auf unterschiedlichen Ebenen analysieren. In einer Mikro-Ebene lassen sich Kontakte zwischen Ego und verschiedenen Alteri analysieren, bei denen auch die Verbindungen der Alteri untereinander erfasst werden. Ein solches Verständnis geht über eine reine Erfassung von sozialen Kontakten hinaus. Auf einer Meso-Ebene können diese Netzwerke für gröBere Gruppen oder kleinräumige Gebiete wie Schulen oder Nachbarschaften gemessen werden. Aus einer solchen Perspektive lassen sich Rückschlüsse über Verbindungen zwischen Netzwerken ziehen. Zentraler Nachteil eines so umfassenden Verständnisses sozialer Netzwerke ist der methodische Aufwand, der zur Erfassung sozialer Netzwerke notwendig ist (siehe Kap. „Netzwerkanalyse“).

Der Aspekt der Messung und Erhebung so umfassend verstandener sozialer Netzwerke ist ein wichtiger Aspekt der vorliegenden Arbeit. Viele der bisherigen Arbeiten, insbesondere im Zusammenhang mit der Untersuchung von gesundheitlichen Ungleichheiten, konnten den Ansprüchen komplexer sozialer Netzwerke nicht genügen, da sie sich häufig auf quantitative Aspekte sozialer Netzwerke (etwa die Anzahl von Kontakten) oder auf soziale Unterstützung ohne Berücksichtigung anderer Mitglieder der sozialen Netzwerke (Alteri) konzentriert haben. Für eine komplexe Analyse sozialer Netzwerke im Zusammenhang mit gesundheitlichen Ungleichheiten fehlen neben einem gemeinsamen begrifflichen Verständnis auch entsprechende Daten. An dieser Stelle soll der vorliegende Sammelband aufzeigen, welche Möglichkeiten sich für die Forschung zu gesundheitlichen Ungleichheiten aus einem komplexeren Verständnis von sozialen Netzwerken ergeben könnten und an welchen „Leerstellen“ bisheriger Forschung mit neuen Erkenntnissen gerechnet werden könnte. 


\section{Leseempfehlungen}

Berkman, L. F., \& Krishna, A. (2014). Social Network Epidemiology. In L.F. Berkman \& I. Kawachi (Hrsg.), Social Epidemiology (S. 234-289). Oxford: University Press. Grundlegende und fundierte Aufbereitung des Themas „Soziale Netzwerke und Gesundheit“, sowohl theoretische Grundlagen als auch empirische Zusammenhänge werden dargestellt.

Henning, M., \& Kohl, S. (2011). Rahmen und Spielräume sozialer Beziehungen. Zum Einfluss des Habitus auf die Herausbildung von Netzwerkstrukturen. Wiesbaden: VS. Gute Übersichtsarbeit zur theoretischen Verortung sozialer Beziehungen.

Kawachi, I., Berkman, L. (2014). Social Cohesion, Social Capital, and Health. In L. Berkman \& I. Kawachi (Hrsg.), Social Epidemiology (S. 290319). Oxford: University Press. Sehr fundierte und ausführliche Übersichtsarbeit zum Thema „Soziales Kapital und Gesundheit“.

\section{Literatur}

Airaksinen, J., Elovainio, M., Hakulinen, C., Elovainio, M., Lehtimäki, T., Raitakari, O. T., Keltikangas-Järvinen, L., \& Jokela, M. (2015). Neighborhood effects in depressive symptoms, social support, and mistrust: Longitudinal analysis with repeated measurements. Social Science and Medicine, 136-137, 10-16.

Barrera, M. (1986). Distinctions between social support concepts, measures and models. American Journal of Community Psychology, 14(4), 413-426.

Berkman, L. F., \& Krishna, A. (2014). Social network epidemiology. In L. F. Berkman \& I. Kawachi (Hrsg.), Social epidemiology (S. 234-289). Oxford: University Press.

Berkman, L. F., \& Syme, S. L. (1979). Social networks, host resistance, and mortality: A nine-year follow-up study of Alameda County residents. American Journal of Epidemiology, 109(2), 186-204.

Berkman, L. F., Glass, T., et al. (2000). From social integration to health: Durkheim in the new millennium. Social Science and Medicine, 51(6), 843-845.

Bollen, K. A., \& Hoyle, R. H. (1990). Perceived cohesion: A conceptual and empirical examination. Social Forces, 69(2), 479-504.

Bourdieu, P. (1994). Die feinen Unterschiede. Kritik der gesellschaftlichen Urteilskraft. Frankfurt a. M.: Suhrkamp.

Brown, R. J., \& Hewstone, M. (2005). An integrative theory of intergroup contact. In M. Zanna (Hrsg.), Advances in experimental psychology (S. 255-331). San Diego: Academic.

Burt, R. S. (1982). Toward a structural theory of action. Network models of social structure, perception and action. New York: Academic. 
Cobb, S. (1976). Social support as a moderator of life stress. Psychosomatic Medicine, 38(5), 300-314.

Coleman, J. S. (1990). Foundations of social theory. Cambridge: The Belknap Press of Harvard University.

Dahl, E., \& Malmberg-Heimonen, I. (2010). Social inequality and health: The role of social capital. Sociology of Health \& Illness, 32(7), 1102-1119.

Durkheim, E. (1993). Der Selbstmord. Frankfurt a. M.: Suhrkamp.

Freeman, L. C. (2004). The development of social network analysis: A study in the sociology of science. Vancouver: Booksurge Publishing.

Fuhse, J. (2008). Netzwerke und soziale Ungleichheit. In C. Stegbauer (Hrsg.), Netzwerkanalyse und Netzwerktheorie (S. 79-90). Wiesbaden: VS.

Gibson, D. (2005). Taking turns and talking ties: Network structure and conversational sequences. American Journal of Sociology, 110(6), 1561-1597.

Granovetter, M. (1973). The strength of weak ties. American Journal of Sociology, 78(6), 1360-1380.

Häußling, R. (2010). Relationale Soziologie. In C. Stegbauer \& R. Häußling (Hrsg.), Handbuch Netzwerkforschung (S. 63-87). Wiesbaden: VS.

Henning, M., \& Kohl, S. (2011). Rahmen und Spielräume sozialer Beziehungen. Zum Einfluss des Habitus auf die Herausbildung von Netzwerkstrukturen. Wiesbaden: VS.

Hewstone, M. (2015). Consequences of diversity for social cohesion and prejudice: The missing dimension of intergroup contact. Journal of Social Issues, 71, 417-438.

Holt-Lunstad, J., Smith, T. B., et al. (2010). Social relationships and mortality risk: A meta-analytic review. PLoS One Medicine, 7(7), e1000316.

Holzer, B. (2009). Netzwerktheorie. In Kneer, G. \& Schroer, M. (Hrsg.), Handbuch Soziologische Theorien (S. 253-276). Wiesbaden: Springer VS.

House, J. S., Landis, K. R., et al. (1988). Social relationships and health. Science, 241(4865), 540-545.

Ichida, Y., Kondo, K., et al. (2009). Social capital, income inequality and self-rated health in Chita peninsula, Japan: A multilevel analysis of older people in 25 communities. Social Science and Medicine, 69(4), 489-499.

Kaplan, B. H., Cassel, J. C., et al. (1977). Social support and health. Medical Care, 15(5), 47-58.

Kawachi, I., Berkman, L. (2014). Social cohesion, social capital, and health. In L.F. Berkman I. Kawachi (Hrsg.), Social epidemiology (S. 290-319). Oxford: University Press.

Laireiter, A. (1993). Soziales Netzwerk und soziale Unterstützung: Konzepte, Methoden und Befunde. Bern: Huber.

Lakey, B. \& Cohen, S. (2000). Social support theory and measurement. In S. Cohen, L. G. Underwood et al. (Hrsg.), Social support measurement and intervention. A guide for health and social scientists (S. 29-52). Oxford: Oxford University Press.

Laurence, J., \& Bentley, L. (2016). Does ethnic diversity have a negative effect on attitudes towards the community? A longitudinal analysis of the causal claims within the ethnic diversity and social cohesion debate. European Sociological Review, 32(1), 54-67.

Lin, N. (2000). Inequality in social capital. Contemporary Sociology, 29(6), 785-795.

Lin, N., Ye, X., et al. (1999). Social support and depressed mood: A structural analysis. Journal of Health and Social Behavior, 40(4), 344-359. 
Lüdicke, J., \& Diewald, M. (2007). Soziale Netzwerke und soziale Ungleichheit. Zur Rolle von Sozialkapital in modernen Gesellschaften. Wiesbaden: VS.

Parsons, T. (1951). The social system. London: Routledge.

Pickett, K. E., \& Pearl, M. (2001). Multilevel analysis of neighbourhood socioeconomic context and health outcomes: A critical review. Journal of Epidemiology and Coтmunity Health, 55(2), 111-122.

Putnam, R. D. (1995). Tuning in, tuning out: The strange disappearance of social capital in America. Political Science and Politics, 28(4), 664-683.

Putnam, R. D. (2007). E pluribus unum: Diversity and community in the twenty-first century. The 2006 Jonathan Skytte Prize Lecture. Scandinavian Political Studies, 30, 137174.

Siegrist, J., \& Wahrendorf, M. (2016). Failed social reciprocity beyond the work role. In J. Siegrist \& M. Wahrendorf (Hrsg.), Work stress and health in a globalized economy. Aligning perspectives on health, safety and well-being (S. 275-291). Cham: Springer.

Turner, R. J., \& Marino, F. (1994). Social support and social structure: A descriptive epidemiology. Journal of Health and Social Behavior, 35(3), 193-212.

Uchino, B. N. (2009). What a lifespan approach might tell us about why distinct measures of social support have differential links to physical health. Journal of Social and Personal Relationships, 26(1), 53-62.

White, C. (1995). Network switchings and Bayesian forks: Reconstructing the social and behavioral sciences. Social Research, 62(4), 1035-1063.

Wills, T. A., \& Shinar, O. (2000). Measuring perceived and received social support. In S. Cohen, L. G. Underwood, et al. (Hrsg.), Social support measurement and intervention - A guide for health and social scientists (S. 86-135). Oxford: Oxford University Press.

Open Access Dieses Kapitel wird unter der Creative Commons Namensnennung 4.0 International Lizenz (http://creativecommons.org/licenses/by/4.0/deed.de) veröffentlicht, welche die Nutzung, Vervielfältigung, Bearbeitung, Verbreitung und Wiedergabe in jeglichem Medium und Format erlaubt, sofern Sie den/die ursprünglichen Autor(en) und die Quelle ordnungsgemäß nennen, einen Link zur Creative Commons Lizenz beifügen und angeben, ob Änderungen vorgenommen wurden.

Die in diesem Kapitel enthaltenen Bilder und sonstiges Drittmaterial unterliegen ebenfalls der genannten Creative Commons Lizenz, sofern sich aus der Abbildungslegende nichts anderes ergibt. Sofern das betreffende Material nicht unter der genannten Creative Commons Lizenz steht und die betreffende Handlung nicht nach gesetzlichen Vorschriften erlaubt ist, ist für die oben aufgeführten Weiterverwendungen des Materials die Einwilligung des jeweiligen Rechteinhabers einzuholen.

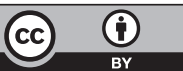

